\title{
Two new criteria for comparison in the Bruhat order
}

\author{
Brian Drake, Sean Gerrish, Mark Skandera \\ Dept. of Mathematics, Brandeis University \\ MS 050, P.O. Box 9110, Waltham, MA 02454 \\ bdrake@math. brandeis.edu \\ Dept. of Mathematics, University of Michigan \\ 2074 East Hall, Ann Arbor, MI 48109-1109 \\ sgerrish@umich.edu \\ Dept. of Mathematics, Dartmouth College \\ 6188 Bradley Hall, Hanover, NH 03755-3551 \\ mark.skandera@dartmouth.edu
}

Submitted: Sep 25, 2003; Accepted: Jan 20, 2004; Published: Mar 31, 2004

MR Subject Classifications: 15A15, 05E05

\begin{abstract}
We give two new criteria by which pairs of permutations may be compared in defining the Bruhat order (of type $A$ ). One criterion utilizes totally nonnegative polynomials and the other utilizes Schur functions.
\end{abstract}

The Bruhat order on $S_{n}$ is often defined by comparing permutations $\pi=\pi(1) \cdots \pi(n)$ and $\sigma=\sigma(1) \cdots \sigma(n)$ according to the following criterion: $\pi \leq \sigma$ if $\sigma$ is obtainable from $\pi$ by a sequence of transpositions $(i, j)$ where $i<j$ and $i$ appears to the left of $j$ in $\pi$. (See e.g. [7, p.119].) A second well-known criterion compares permutations in terms of their defining matrices. Let $M(\pi)$ be the matrix whose $(i, j)$ entry is 1 if $j=\pi(i)$ and zero otherwise. Defining $[i]=\{1, \ldots, i\}$, and denoting the submatrix of $M(\pi)$ corresponding to rows $I$ and columns $J$ by $M(\pi)_{I, J}$, we have the following.

Theorem 1 Let $\pi$ and $\sigma$ be permutations in $S_{n}$. Then $\pi$ is less than or equal to $\sigma$ in the Bruhat order if and only if for all $1 \leq i, j \leq n-1$, the number of ones in $M(\pi)_{[i],[j]}$ is greater than or equal to the number of ones in $M(\sigma)_{[i],[j]}$.

(See [1], [2], [3], [6, pp. 173-177], [8] for more criteria.) Using Theorem 1 and our defining criterion we will state and prove the validity of two more criteria.

Our first new criterion defines the Bruhat order in terms of totally nonnegative polynomials. A matrix $A$ is called totally nonnegative (TNN) if the determinant of each square submatrix of $A$ is nonnegative. (See e.g. [5].) A polynomial in $n^{2}$ variables $f\left(x_{1,1}, \ldots, x_{n, n}\right)$ is called totally nonnegative (TNN) if for each TNN matrix $A=\left(a_{i, j}\right)$ 
the number $f\left(a_{1,1}, \ldots, a_{n, n}\right)$ is nonnegative. Some recent interest in TNN polynomials is motivated by problems in the study of canonical bases. (See [10].)

Theorem 2 Let $\pi$ and $\sigma$ be two permutations in $S_{n}$. Then $\pi$ is less than or equal to $\sigma$ in the Bruhat order if and only if the polynomial

$$
x_{1, \pi(1)} \cdots x_{n, \pi(n)}-x_{1, \sigma(1)} \cdots x_{n, \sigma(n)}
$$

is totally nonnegative.

Proof: $\quad(\Rightarrow)$ If $\pi=\sigma$ then (1) is obviously TNN. Suppose that $\pi$ is less than $\sigma$ in the Bruhat order. If $\pi$ differs from $\sigma$ by a single transposition $(i, j)$ with $i<j$, then we have $\pi(i)=\sigma(j)<\pi(j)=\sigma(i)$, and the polynomial (1) is equal to

$$
\frac{x_{1, \pi(1)} \cdots x_{n, \pi(n)}}{x_{i, \pi(i)} x_{j, \pi(j)}}\left(x_{i, \pi(i)} x_{j, \pi(j)}-x_{i, \pi(j)} x_{j, \pi(i)}\right)
$$

which is clearly TNN. If $\pi$ differs from $\sigma$ by a sequence of transpositions, then the polynomial (1) is equal to a sum of polynomials of the form (2) and again is TNN.

$(\Leftarrow)$ Suppose that $\pi$ is not less than or equal to $\sigma$ in the Bruhat order. By Theorem 1 we may choose indices $1 \leq k, \ell \leq n-1$ such that $M(\sigma)_{[k],[\ell]}$ contains $q+1$ ones and $M(\pi)_{[k],[\ell]}$ contains $q$ ones. Now define the matrix $A=\left(a_{i, j}\right)$ by

$$
a_{i, j}= \begin{cases}2 & \text { if } i \leq k \text { and } j \leq \ell \\ 1 & \text { otherwise }\end{cases}
$$

It is easy to see that $A$ is TNN, since all square submatrices of $A$ have determinant equal to 0,1 , or 2 . Applying the polynomial (1) to $A$ we have

$$
a_{1, \pi(1)} \cdots a_{n, \pi(n)}-a_{1, \sigma(1)} \cdots a_{n, \sigma(n)}=-2^{q},
$$

and the polynomial (1) is not TNN.

Our second new criterion defines the Bruhat order in terms of Schur functions. (See [9, Ch.7] for definitions.) Any finite submatrix of the infinite matrix $H=\left(h_{j-i}\right)_{i, j \geq 0}$, where $h_{k}$ is the $k$ th complete homogeneous symmetric function and $h_{k}=0$ for $k<0$, is called a Jacobi-Trudi matrix. Let us define a polynomial in $n^{2}$ variables $f\left(x_{1,1}, \ldots, x_{n, n}\right)$ to be Schur nonnegative (SNN) if for each Jacobi-Trudi matrix $A=\left(a_{i, j}\right)$ the symmetric function $f\left(a_{1,1}, \ldots, a_{n, n}\right)$ is equal to a nonnegative linear combination of Schur functions. Some recent interest in SNN polynomials is motivated by problems in algebraic geometry [4, Conj. 2.8, Conj. 5.1].

Theorem 3 Let $\pi$ and $\sigma$ be permutations in $S_{n}$. Then $\pi$ is less than or equal to $\sigma$ in the Bruhat order if and only if the polynomial

$$
x_{1, \pi(1)} \cdots x_{n, \pi(n)}-x_{1, \sigma(1)} \cdots x_{n, \sigma(n)}
$$

is Schur nonnegative. 
Proof: $\quad(\Rightarrow)$ If $\pi=\sigma$ then (3) is obviously SNN. Let $A$ be an $n \times n$ Jacobi-Trudi matrix and suppose that $\pi$ is less than $\sigma$ in the Bruhat order. If $\pi$ differs from $\sigma$ by a single transposition $(i, j)$, then for some partition $\nu$ and some $k, \ell, m(\ell, m>0)$, the evaluation of the polynomial (3) at $A$ is equal to

$$
h_{\nu}\left(h_{k+\ell} h_{k+m}-h_{k+\ell+m} h_{k}\right),
$$

and (3) is clearly SNN. If $\pi$ differs from $\sigma$ by a sequence of transpositions, then the evaluation of (3) at $A$ is equal to a sum of polynomials of the form (4) and again (3) is SNN.

$(\Leftarrow)$ Suppose that $\pi$ is not less than or equal to $\sigma$ in the Bruhat order. By Theorem 1 we may choose indices $1 \leq k, \ell \leq n-1$ such that $M(\sigma)_{[k],[\ell]}$ contains $q+1$ ones and $M(\pi)_{[k],[\ell]}$ contains $q$ ones. Now define the nonnegative number $r=(k-q)(n+k-\ell-2)$ and consider the Jacobi-Trudi matrix $B$ defined by the skew shape $(n-1+2 r)^{k}(n-1+r)^{n-k} / r^{\ell}$,

$$
B=\left[\begin{array}{cccccc}
h_{n-1+r} & \cdots & h_{n+\ell-2+r} & h_{n+\ell-1+2 r} & \cdots & h_{2 n-2+2 r} \\
\vdots & & \vdots & \vdots & & \vdots \\
h_{n-k+r} & \cdots & h_{n-k+\ell-1+r} & h_{n-k+\ell+2 r} & \cdots & h_{2 n-k-1+2 r} \\
h_{n-k-1} & \cdots & h_{n-k+\ell-2} & h_{n-k+\ell-1+r} & \cdots & h_{2 n-k-2+r} \\
\vdots & & \vdots & \vdots & & \vdots \\
h_{0} & \cdots & h_{\ell-1} & h_{\ell+r} & \cdots & h_{n-1+r}
\end{array}\right] .
$$

The polynomial (3) applied to $B$ may be expressed as $h_{\lambda}-h_{\mu}$ for some appropriate partitions $\lambda, \mu$ depending on $\pi, \sigma$, respectively. We claim that $\lambda$ is incomparable to or greater than $\mu$ in the dominance order. Since $M(\pi)_{[k],[\ell+1, n]}$ contains $k-q$ ones we have that

$$
\lambda_{1}+\cdots+\lambda_{k-q} \geq(k-q)(n-k+\ell+2 r) .
$$

Similarly, we have

$$
\mu_{1}+\cdots+\mu_{k-q} \leq(k-q-1)(2 n-2+2 r)+\max \{n+\ell-2+r, 2 n-k-2+r\} .
$$

Subtracting (6) from (5), we obtain

$$
\left(\lambda_{1}+\cdots+\lambda_{k-q}\right)-\left(\mu_{1}+\cdots+\mu_{k-q}\right) \geq n-\max \{\ell, n-k\}>0,
$$

as desired.

Recall that the Schur expansion of $h_{\mu}$ is

$$
h_{\mu}=s_{\mu}+\sum_{\nu>\mu} K_{\nu, \mu} s_{\nu}
$$

where the comparison of partitions $\nu>\mu$ is in the dominance order and the nonnegative Kostka numbers $K_{\nu, \mu}$ count semistandard Young tableaux of shape $\nu$ and content $\mu$. (See e.g. [9, Prop. 7.10.5, Cor. 7.12.4].) It follows that the coefficient of $s_{\mu}$ in the Schur expansion of $h_{\lambda}-h_{\mu}$ is -1 and the polynomial (3) is not SNN.

The authors are grateful to Sergey Fomin, Zachary Pavlov, Alex Postnikov, Christophe Reutenauer, Brendon Rhoades, Richard Stanley, John Stembridge, and referees for helpful conversations. 


\section{References}

[1] A. BJöRneR. Orderings of Coxeter groups. In Combinatorics and Algebra (C. Greene, ed.), vol. 34 of Contemp. Math.. American Mathematical Society, Providence, RI, 1984 pp. 175-195.

[2] A. Buörner and F. Brenti. An improved tableau criterion for Bruhat order. Electron. J. Combin., 3 (1996). Research paper 22, 5 pp. (electronic).

[3] V. Deodhar. Some characterizations of Bruhat ordering on a Coxeter group and determination of the relative Möbius function. Inventiones Math., 39 (1977) pp. $187-198$.

[4] S. Fomin, W. Fulton, C. Li, And Y. Poon. Eigenvalues, singular values, and Littlewood-Richardson coefficients, 2003. Preprint math.AG/03013078 on ArXiv.

[5] S. Fomin And A. Zelevinsky. Total positivity: Tests and parametrizations. Math. Intelligencer, 22 (2000) pp. 23-33.

[6] W. Fulton. Young Tableaux; With Applications to Representation Theory and Geometry, vol. 35 of London Mathematical Society Student Texts. Cambridge University Press, New York, 1997.

[7] J. E. Humphreys. Reflection groups and Coxeter groups. Cambridge University Press, 1990.

[8] A. Lascoux and M. P. Schützenberger. Treillis et bases des groupes de Coxeter. Electron. J. Combin., 3 (1996). Research paper 27, 35 pp. (electronic).

[9] R. Stanley. Enumerative Combinatorics, vol. 2. Cambridge University Press, Cambridge, 1999.

[10] A. Zelevinsky. From Littlewood-Richardson coefficients to cluster algebras in three lectures. In Symmetric Functions 2001: Surveys of Developments and Perspectives (S. Fomin, ed.), vol. 74 of NATO Science Series II: Mathematics, Physics, and Chemistry. Kluwer, Dordrecht, 2002 pp. 253-273. 